The Journal of Animal \& Plant Sciences, 31(4): 2021, Page: 1101-1106

ISSN (print): 1018-7081; ISSN (online): 2309-8694

\title{
GENETIC DIVERSITY OF MUNGBEAN GENOTYPES WITH DIFFERENT RESISTANCE AGAINST MUNGBEAN YELLOW MOSAIC VIRUS DETERMINED BY MICRO SATELLITE MARKERS
}

\author{
R. Binyamin ${ }^{1}$, M.A. Khan ${ }^{2}$, F. S.Awan 3 , S. Ali ${ }^{2}$, M.A. Zeshan ${ }^{* 4}$, A. Masroor ${ }^{1}$, N. Ahmed ${ }^{5}$ and M.U. Ghani ${ }^{6}$ \\ ${ }^{1}$ University of Agriculture Faisalabad, Sub-Campus Burewala-Verhari, Pakistan. \\ ${ }^{2}$ Department of Plant Pathology, University of Agriculture Faisalabad, Pakistan. \\ ${ }^{3}$ Centre of Agricultural Biochemistry and Biotechnology, University of Agriculture Faisalabad, Pakistan. \\ ${ }^{4}$ Department of Plant Pathology, College of Agriculture, University of Sargodha. Pakistan. \\ ${ }^{5}$ Department of Plant Pathology, Muhammad Nawaz Sharif University of Agriculture, Multan. Pakistan. \\ ${ }^{6}$ Institute of Soil and Environmental Sciences, University of Agriculture Faisalabad, Pakistan. \\ Correspondence E-mail: muhammad.ahmad@uos.edu.pk
}

\begin{abstract}
Mungbean yellow mosaic virus (MYMV) is a single-stranded DNA (ssDNA) begomovirus that is the most prevalent threats in Pakistan to mungbean crop. Ten genotypes were selected from 127 genotypes which have already been screened for resistance under field conditions. A total of 38 bands were scored, exhibited high level of polymorphism i.e., $68.42 \%$, with amplification range of (1-7bands). Genetic analysis often primers revealed similarities in the range of 66.60-97.70\%. Majority of primers were moderately informative with polymorphism information content (PIC) value $(0.00-0.566)$ and average PIC value of all primers was 0.379 . Cluster analysis inferred that genotypes showing resistant response against MYMV were present in un-clustered form in dendrogram. On the basis of phylogenetic analysis NM-2011 has higher genetic difference which reduces to the next branches showed that the susceptible (S) and moderately susceptible (MS) genotypes diverged from resistant genotypes. Diverse field response of mungbean was confirmed even on SSR markers and furthermore it is recommended that molecular markers are effective tags for genetic diversity calculation in mungbean germplasm and could be utilized for the future breeding program regarding mungbean crop.
\end{abstract}

Keywords: Mungbean, similarity index, dendrogram, MYMV, Resistance, SSR markers

https://doi.org/10.36899/JAPS.2021.4.0307

Published online December 18, 2020

\section{INTRODUCTION}

Mungbean (Vigna radiate L.) was originated from Persia (Iran) and brought to Northwest and South part of Indian sub-continent (Fuller, 2007). It is major legume crop which is rich source of minerals, vitamins, and proteins with very short growth period in summer and spring seasons (Haytowitz and Matthews,1986; Akhtar et al. 2009). Mungbean in combination with bacteria in soil fixes nitrogen, and increases tolerance of the crop against drought. Mungbean yellow mosaic virus (MYMV) causes huge losses in the production of mungbean in Pakistan, India, Bangladesh and SriLanka (Qazi et al., 2007; Biswass et al. 2008; John et al. 2008). MYMV causes 5$100 \%$ losses depending on cultivar resistance level, pathogen virulence and vector's population (Nene, 1972; Singh, 1980; Rathi, 2002). The molecular genetics and breeding of mungbean always remained neglected area of research. Determination of genetic variation is a fundamental need in understanding the evolutionary and genetic association of different accessions for the selection of specific genotype and for the development of important breeding approaches for disease management (Lavanya et al. 2008). Number of researchers determined the genetic diversity of mungbean (Kumar et al. 2002; Dikshit et al. 2007; Singh et al. 2012; Wang et al. 2012; Binyamin et al. 2016) but its genetic design is yet poorly understood. For genetic diversity determination, simple sequence repeats (SSR) markers proved effective as SSR results are reproducible. A number of commercial mungbean cultivars are susceptible to MYMV infection. Therefore, it is utmost need to determine the markers which have close relationship to MYMV resistance genes. The resistant genes could be in corporate into the cultivars which have great yield potential by using the marker-based breeding. Breeding processes which are phenotypic based take more time while application of molecular markers is more rapid and trust worthy for resistance breeding. Besides diversity research, markers can also be used for determination of agronomic traits (Azmat and Khan, 2010) and resistant genes (Michelmore et al. 1991). The present research is focused upon the practice of SSR markers to check relationship in genetic diversity of mungbean germplasm and resistance against MYMV. The genotypes used in this study are being evaluated first time in Pakistan on molecular basis. The study provides the basic knowledge regarding aspects of the mungbean genotypes, 
phylogenetic relationship and intra specific diversity that would be helpful in the development of resistant cultivars against MYMV.

\section{MATERIALS AND METHODS}

Mungbean genotype DNA extraction: In 2015, 127 genotypes were screened against MYMV under field conditions (Binyamin et al. 2015), out of which ten candidates mungbean genotypes were selected for molecular studies (Table1).

All the molecular research work has been carried out in 2015-16 at "Genomics and Finger printing Lab." Centre of Agricultural Biochemistry and Biotechnology, University of Agriculture Faisalabad. The selected genotypes were further analyzed to determine their genetic diversity by using SSR markers (Table2).

The seeds of cultivars (NM-2011, NM-2006, Azri-06, NM-92, C294-4-36, NM-54, M-6, 8008, 8010, and 8011) were grown under controlled conditions at $30^{\circ} \mathrm{C}$, humidity $75 \%$, light and darkness $12: 12$ hours. At the time of harvest, fresh leaf samples (8-10) were collected from mungbean plants which were potted and genomic DNA was isolated from these samples following cetyl trimethyl ammonium bromide (CTAB) procedure (Doyle and Doyle, 1990), with minor amendments. Nano drop spectrophotometer 2000 (Thermo Scientific, Germany) was used to determine the DNA concentration and dilutions were made on the basis of the best amplification.

Table1: List of mungbean genotypes used for simple sequence repeats (SSR) markers analysis.

\begin{tabular}{cllccl}
\hline Sr. No. & Varieties/lines & Resistance level & Sr. No. & Varieties/lines & Resistance level \\
\hline 1 & NM-2011 & Resistant & 6 & NM-54 & Moderately susceptible \\
2 & NM-2006 & Resistant & 7 & M-6 & Moderately susceptible \\
3 & Azri-06 & Resistant & 8 & 8008 & Highly susceptible \\
4 & NM-92 & Moderately resistant & 9 & 8010 & Highly susceptible \\
5 & C $_{2} 94-4-36$ & Moderately resistant & 10 & 8011 & Highly susceptible \\
\hline
\end{tabular}

Table2: SSR primers used in the present study.

\begin{tabular}{|c|c|c|c|c|c|}
\hline $\begin{array}{l}\text { Sr. } \\
\text { No. }\end{array}$ & $\begin{array}{c}\text { Primers } \\
\text { Name }\end{array}$ & Sequence $\left(5^{\prime} \rightarrow 3^{\prime}\right)$ & $\begin{array}{l}\text { No. of } \\
\text { alleles }\end{array}$ & $\begin{array}{l}\text { PIC } \\
\text { Value }\end{array}$ & $\begin{array}{c}\text { Polymorphism } \\
\text { (\%) }\end{array}$ \\
\hline \multirow{2}{*}{1} & VR044F & CCCATGAAGGTATGAGACAACA & \multirow[b]{2}{*}{1} & \multirow{2}{*}{0} & \multirow{2}{*}{0} \\
\hline & VR044R & GACTGAGAAAGAGAGAGAAGCATTT & & & \\
\hline \multirow{2}{*}{2} & VR0222F & ТСТСТТСТСТСТТСТСТСТTСТTСТTC & \multirow{2}{*}{3} & \multirow{2}{*}{0.444} & \multirow{2}{*}{66.66} \\
\hline & VR0222R & TTGTGTCTGAGGCTATGTTGGT & & & \\
\hline \multirow{2}{*}{3} & VR0223F & GCGTGATCGAGGCAGACTAT & \multirow{2}{*}{1} & \multirow{2}{*}{0} & \multirow{2}{*}{0} \\
\hline & VR0223R & GTGGGTAGCTCGGTAATAGCAC & & & \\
\hline \multirow{2}{*}{4} & LR7319BF & CTGCTTTTTGGGGATTTCAG & \multirow{2}{*}{7} & \multirow{2}{*}{0.428} & \multirow{2}{*}{71.42} \\
\hline & LR7319BR & CACGCAAACAGAAAGCAGAG & & & \\
\hline \multirow{2}{*}{5} & LR7322BF & TCAGTCAGTGTCGATAGCATAGC & \multirow{2}{*}{6} & \multirow{2}{*}{0.416} & \multirow{2}{*}{66.66} \\
\hline & LR7322BR & GACACAGAGAGAGAGAGAGAG & & & \\
\hline \multirow{2}{*}{6} & LR7323AF & TGACGGAGAGAGAGAGAGAGAG & \multirow{2}{*}{3} & \multirow{2}{*}{0.388} & \multirow{2}{*}{66.66} \\
\hline & LR7323AR & TGCTTCCTTTTGTCTGAGTTAGAA & & & \\
\hline \multirow{2}{*}{7} & LR7323BF & GCTATGCTATCGACACTGACTGA & \multirow{2}{*}{4} & \multirow{2}{*}{0.541} & \multirow{2}{*}{75.00} \\
\hline & LR7323BR & GCGCAAAGAGAGAGAGAGAGA & & & \\
\hline \multirow{2}{*}{8} & LR7315AF & GTAGCGCAGAGAGAGAGAGAG & \multirow[b]{2}{*}{3} & \multirow{2}{*}{0.444} & \multirow{2}{*}{66.66} \\
\hline & LR7315AR & CAAAACGGCTCATTCAGCTT & & & \\
\hline \multirow{2}{*}{9} & LR738AF & CGCAAAGAGAGAGAGAGAG & \multirow{2}{*}{5} & \multirow{2}{*}{0.566} & 8000 \\
\hline & LR738AR & СССССАТCTGAAAGAAAGAG & & & \\
\hline 10 & LR733BF & GAGAGCAACGATTGAAAAATG & 5 & 0566 & 8000 \\
\hline & LR733BR & GTTCGTAGTTACATTGTCCC & & & \\
\hline & & & 3.8 & 0.3793 & 57.04 \\
\hline
\end{tabular}

Primer sequence of SSR markers: For genetic characterization of ten mungbean genotypes fifteen SSR primers were used. Based on previous literature, SSR primers were chosen on the basis of their ability of amplification of DNA and polymorphism. 
PCR conditions for SSR markers: The polymerase chain reaction (PCR) was conducted in PCR thermalcycler (AG No.533300839, Germany) by using volume of $20 \mu \mathrm{L}$. All PCR amplified products were run on agarose gelelectrophoresis chamber with $1 \%(\mathrm{w} / \mathrm{v})$ agarose gelat
$80 \mathrm{~V}$ for 1 hour, ethidium bromide was used for staining, UV transilluminator was used for visualization at $300 \mathrm{~nm}$ and captured the photo by using geldocs of tware system (SynGen, Synoptics Ltd, UK).

Table3:Informativeness of SSR markers among the Mungbean genotypes.

\begin{tabular}{lccc}
\hline & \multicolumn{3}{c}{ Ranges of PIC Values } \\
\hline \multicolumn{1}{c}{ Information level } & $\leq 0.30$ Uninformative & $0.30-0.50$ ModeratelyInformative & $\geq 0.50$ HighlyInformative \\
No. of Primers & 02 & 05 & 03 \\
$\%$ age of Primers & $20.00 \%$ & $50.00 \%$ & $30.00 \%$ \\
\hline
\end{tabular}

Data analysis: The visibly resolved bands were noted and absence orpresence was counted by 0 and 1 , respectively. For the estimation of molecular weight of bands, DNA ladder was used. For further analysis data for reproducible bands were utilized. PopGene 32 software (version1.44); (Yeh et al. 2000) was used to create a similarity matrix by conducting multivariate analysis which is based on Nei's Un-weighted Paired Group of Arithmetic Means Average (UPGMA) to estimate the relatedness and genetic distance of mungbean germplasm. The polymorphism information content (PIC) value was calculated with equation formulated by Botstein et al. (1980):

$$
\mathrm{PIC}=1-\sum_{i=1}^{n} p_{i}^{2}-2 \sum_{i=1}^{n-1} \sum_{j=i+1}^{n} p_{i}^{2} p_{j}^{2}=1-\sum_{i=1}^{n} p_{i}^{2}-\left(\sum_{i=1}^{n} p_{i}^{2}\right)^{2}+\sum_{i=1}^{n} p_{i}^{4},
$$

\section{RESULTS}

Ten mungbean genotypes were chosen because of their different behaviors against MYMV and were genetically evaluated by fifteen SSR primers. Ten SSR primers were selected for final analysis out of fifteen primers which produced the clear, distinct, and readily detectable fragments having high rate of polymorphism (Table2). Significantly polymorphic results were obtained by using the primers for genetic diversity in mungbean germplasm. The highest total number of bands (7) produced by primer LR $7319 \mathrm{~B}$, followed by primer LR 7322 B (6bands). The highest polymorphic bands were obtained by primer LR 7319B (5bands) followed by LR 7322B, LR738A and LR738B (4bands). Monomorphic banding pattern was produced by using VR 0223, and VR 044 primers (Figure 1). Considering all the primers amplification in total tested genotypes, 38 bands were amplified in PCR reactions. Ten primers generated 26 polymorphic fragments, with maximum polymorphism $(80.00 \%)$ given by LR $738 \mathrm{~B}$, and minimum $(66.00 \%)$ by primers LR7322B, LR7323A, LR7315A, and VR0222 (Table 2). Average numbers of total bands per primer were 3.8 , whereas polymorphic bands average amplification of each primer was 2.6 (bands). Amplified fragments per genotype varied from 28 to 35 , with an average score of 32.20 fragments per genotype. The highest number of fragments were observed in genotype NM-92 (35) and minimum (28) were formed by genotype 8008 (Figure 2).

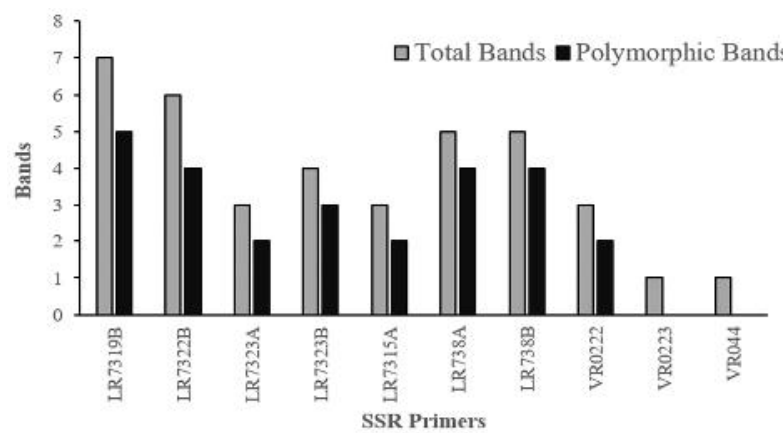

Figure.1:Representing the total number of polymorphic bands obtained per Simple sequence repeats (SSR) markers.

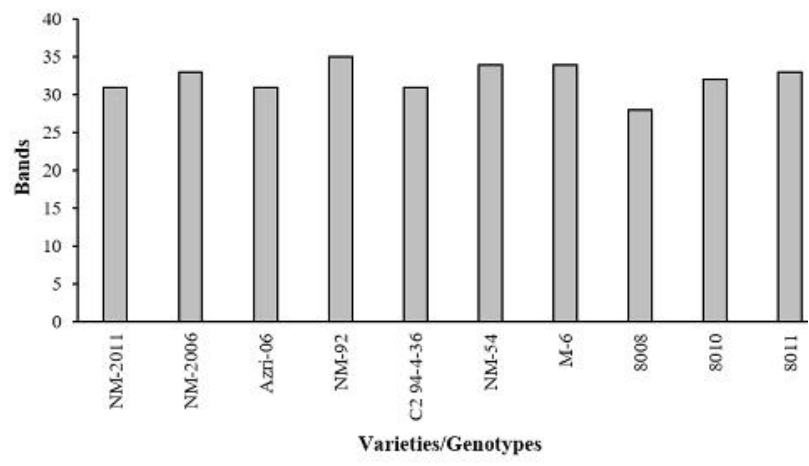

Figure.2:Total number of bands acquired per varieties/genotype.

Polymorphism information content (PIC) value often primers ranges from 0.00 to 0.566 , with an average PIC value of 0.379 . 
Multivariate analysis was performed to produce a similarity matrix based on Nei's UPGMA for estimation of genetic distance and relatedness of mungbean genotypes. Dendrogram showed the similarity coefficient (66.60-97.70\%) within tested mungbean genotypes. Genotypes 8008 and Azri-06 depicted low similarity $(66.60 \%)$, while NM-54 and NM-92 showed maximum resemblance (97.70\%) (Table4).

Table4: Similarity matrix of mungbean genotypes through SSR markers.

\begin{tabular}{|c|c|c|c|c|c|c|c|c|c|c|}
\hline ID & NM-92 & $\begin{array}{l}\text { NM- } \\
2006\end{array}$ & M-6 & 8011 & $\begin{array}{c}\text { C2-94- } \\
4-36\end{array}$ & $\begin{array}{c}\text { NM- } \\
54\end{array}$ & 8008 & Azri-06 & $\begin{array}{l}\text { NM- } \\
2011\end{array}$ & 8010 \\
\hline NM-92 & ***** & 0.8020 & 0.9367 & 0.9464 & 0.9022 & 0.9870 & 0.9860 & 0.8560 & 0.8887 & 0.9623 \\
\hline NM-2006 & 0.2328 & $* * * *$ & 0.7681 & 0.8987 & 0.7787 & 0.8426 & 0.8317 & & 0.7233 & 0.8421 \\
\hline M-6 & 0.0771 & 0.2783 & & 0.8660 & 0.8652 & 0.9045 & & & & 0.9193 \\
\hline 8011 & 0 . & 0.1270 & 0.1458 & ${ }^{* * * *}$ & 0.8990 & 0.9 & & & & 0.8980 \\
\hline C2-94 & 0.1041 & 0.2364 & 0.1448 & 0.1168 & $* * * *$ & 0.8804 & 0.9 & & 0.7790 & 0.8947 \\
\hline NM-5 & 0.0240 & 0.1850 & 0.1003 & 0.0440 & 0.1274 & $* * * *$ & 0.9640 & 0.83600 .888 & 0.9384 & \\
\hline 8008 & 0.0251 & 0.1981 & 0.0536 & 0.0922 & 0.0786 & 0.0486 & $* * * *$ & 0.6750 & 0.8786 & 0.9857 \\
\hline Azri-0 & 0.1792 & 0.3523 & 0.2430 & 0.2440 & 0.1272 & 0.1920 & 0.1448 & $* * * *$ & 0.6990 & 0.8970 \\
\hline NM-2011 & 0.1060 & 0.3428 & 0.1643 & 0.1634 & 0.2588 & 0.1389 & 0.1429 & 0.3737 & & 0.8446 \\
\hline 8010 & 0.0500 & 0.1729 & 0.0851 & 0.1277 & 0.1212 & 0.0743 & 0.0267 & 0.1282 & 0.1827 & \\
\hline
\end{tabular}

Nei's genetic identity (above diagonal) and genetic distance (below diagonal).

Cluster analysis classified 4 (NM-54, NM-92, 8008 and M-06) genotypes into one main clade, while other 6 mungbean genotypes were un-clustered and revealed different behaviors from them $\mathrm{a}$ in clade. The highest level of divergence was observed in genotype NM2011 from them a in clade and it exhibited different behavior from other genotypes (Figure 3). In phylogenetic tree the varieties with largest distance were resistant to MYMV while other varieties were moderate to highly susceptible. At the base of phylogenetic tree, the greater genetic distance of NM-2011 which regularly reduces to the next branches, showed that susceptible and moderately susceptible genotypes may be out come of development from resistant genotypes.

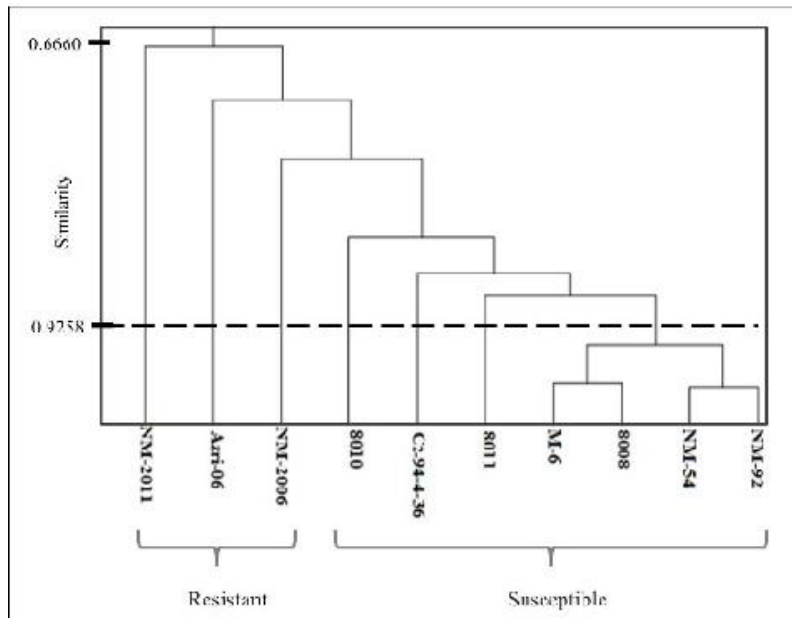

Fig.3: Dendrogram representing the grouping of different genotypes which were relate with different level of resistant genotypes.

\section{DISCUSSION}

To estimate, use and sustain the distinction in a germplasm, it is necessary to determine the range of genetic diversity a mong genotypes (Mohammadi and Prasanna, 2003). In a previous study SCAR marker have been employed to determine the molecular markers of MYMV resistance gene (Binyamin et al., 2015). Microsatellites markers are co-dominant markers and have more reproducibility as compared to random amplified polymorphic DNA (RAPD) markers. So, SSR markers are more appropriate for study of genetic diversity (Brondani et al. 2005). For tagging of varietal identification SSR markers were used ( $\mathrm{Li}$ et al. 2000). Keeping in view the advantages of SSR markers as described above, in current study they were used mainly for the estimation of mungbean genotypes genetic diversity. In a previous study, number of alleles which ranged from 2-6 was observed among genetic variation in mungbean germplasm (Gupta et al.2013). The findings were parallel to current results as maximum number (seven) of loci were amplified by SSR marker LR 7319B, while single loci amplification was produced by VR0223 and VR044 markers. Another study of mungbean genetic diversity depicted that 1-2 alleles were amplified by each primer (Singh et al. 2012), which were less than results of current study (one to seven), and polymorphism level was greater than current study. The number of fragments amplified in current study was higher than reported in a study of mungbean germplasm genetic diversity (Kumar et al., 2002).

Polymorphism information content (PIC) value gives an estimate of the discriminatory power of a locus, by counting the number of alleles and relative frequencies 
of these alleles. PIC values $(0.00-0.566)$ showed more informative nature of ten primers as compared to Chattopadhyay et al. (2005) who reported PIC value range (0.0-0.5) among 80 mungbean genotypes. These results are comparable with Gwag et al. (2010) who reported PIC value range (0.080-0.544) of 15SSR markers among 692mungbean genotypes. Tangphatsornruang et al. (2009) reported PIC value range (0.055-0.690) among 17 mungbean genotypes.

The similarity matrix exhibited that Arzi-06 and 8008 genotypes gave maximum divergence, which showed $66.60 \%$ resemblance in their genetic structure. NM-92 and NM-54 exhibited the maximum resemblance $97.70 \%$, which indicate their evolution from a common ancestor. Greater genetic similarity (77-100\%) was observed in mutant mungbean lines, which depicted that these genotypes had less genetic base (Singh et al. 2012), while the mungbean genotypes have very low diversity in present study. The exotic or wild type mungbean germplasm should be merged in the breeding programs to extend the genetic base.

NM-2011 genotype resistant to MYMV was detected in the $1^{\text {st }}$ hypothetical taxonomic unit to be developed from its wild relatives domesticated by continuous selection for its advantage. The three moderately susceptible genotypes (M-6, NM-54, and NM92 ), genetically resemble the highly susceptible genotypes (8008) have developed from similar lines which were frequently used in programs of breeding.

Three resistant varieties remained failed to make a single group cluster which means that they are different from each other and they may have diverse resistance genes that contribute for resistance against MYMV. Present genotypes gave dissimilar behavior against MYMV and after SSR markers the results verified that these exhibits diverse genetic basis.

Based on SSR analysis it is concluded that genotypes during field conditions produced resistant response and depicts diverse genetic make-up and this could be utilized easily for mungbean crop breeding program in future. SSR primers deployed in present study expected to be very useful tool for DNA fingerprinting of closely related mungbean genotypes. These outcomes are highly useful, effective, and fruitful to search for less expensive and workable decisions as no chemical and biological means has been exist for the better management of MYMV issue in Pakistan.

\section{REFERENCES}

Akhtar, K.P., R. Kitsanachandee, P. Srinives, G. Abbas, M.J. Asghar, T.M. Shah, B.M. Atta, O. Chatchawankanphanich, G. Sarwar, M. Ahmad, and N. Sarwar (2009). Field evaluation of mungbean recombinant inbred lines against mungbean yellow mosaic disease using new disease scale in Thailand. Plant Path. J. 25: 422428.

Azmat, M.A. and A. Khan (2010). Assessment of genetic diversity among the varieties of Gossypium arboreum and Gossypium hirsutum through random amplification of polymorphic DNA (RAPD) and simple sequence repeat (SSR) markers. Pakistan J. Bot. 42: 3173-3181.

Binyamin, R., M.A. Khan, I.A. Khan, F.S. Awan, N.A. Khan, and S. Akhtar (2016). Genetic diversity of mungbean genotypes in relation to resistance against mungbean yellow mosaic virus. Pakistan J. Bot. 48: 1273-1277.

Binyamin, R., M.A. Khan, N.A. Khan and I.A. Khan (2015). Application of SCAR markers linked with mungbean yellow mosaic virus diseaseresistance gene in Pakistan mungbean germplasm. Genet. Mol. Res. 14: 2825-2830.

Biswass, K.K. V.G. Malathi, and A. Varma (2008). Diagnosis of symptomless yellow mosaic begomovirus infection in pigeon pea by using cloned mungbean yellow mosaic India virus a probe. J. Plant Biochem. Biotechnol. 17: 9-14.

Botstein, D., L.W. Raymond, M. Skolnick, and R.W. Davis (1980). Construction of a genetic linkage map in man using restriction fragment length polymorphisms. Am. J. Hum. Genet. 32: 314331.

Brondani, R.P. M.I. Zucchi, C. Brondani, P.H. Rangel, T.C. Borda, P.N. Rangel, M.R. Magalhaes, and R. Vencovsky (2005). Genetic structure of wild rice Oryza gluaepatula populations in three Brazilian biomes using microsatellite markers. Genet. 125: 115-123.

Chattopadhyay, K., M.N. Ali, H.K. Sarkar, N. Mandai, and S. Bhattacharyya (2005). Diversity analysis by RAPD and ISSR markers among the selected mungbean (Vigna radiata (L.) Wilczek] genotypes. Ind. J. Genet. Plant Breed. 65(3): 173175.

Dikshit, H.K.T. Jhang, N.K. Singh, K.R. Koundal, K.C. Bansal, N. Chandra, J.L. Tickoo, and T.L. Sharma (2007). Genetic differentiation of Vigna species by RAPD, URP and SSR markers. Biol. Plant. 51: 451-457.

Doyle, J.J. and J.L. Doyle (1990). Isolation of plant DNA from fresh tissue. Focus.12: 13-15.

Fuller, D.Q. (2007). Contrasting patterns in crop domestication and domestication rates: recent archaeo botanical insights from the old world. Ann. Bot.,100: 903-924.

Gupta,S.,D.S.Gupta,K.T.Anjum,A.Pratap,andJ.Kumar(20 13).Transferabilityofsimplesequencerepeatmark ersinblackgram(VignamungoL.Hepper).Aust.J.C ropSci.7:345-353. 
Gwag, J.,D. Anupam, P. Yong-Jin, M. Kyung-Ho, K. Soon-Jae, C. Gyu-Taek, L.Gi-An, L.Sok-Young, K. Hee-Kyoung, and L.Suk-Ha (2010). Assessment of genetic diversity and population structure in mungbean. Gene. Genom. 32(4): 299-308.

Haytowitz, O.B. and R.H. Matthews (1986). Composition of foods, legumes and legume products. United States Department of Agriculture. Agriculture Hand Book, USA.

John, P., P.N. Sivalingam, Q.M. Haq, and N. Kumar (2008). Cowpea golden mosaic disease in Gujrat is caused by a mungbean yellow mosaic India virus isolate with a DNA B variant. Arch. Virol. 153: $1359-1365$.

Kumar, S.V.,G. Tan, G.C. Quah, and K. Yusoff (2002). Isolation of microsatellite markers in mungbean, Vigna radiata. Mol. Ecol. 2: 96-98.

Lavanya, G.R.,J. Srivastava, and S.A. Ranade (2008). Molecular assessment of genetic diversity in mungbean germplasm. J. Genet., 87: 65-74.

Li, J.Z.,P. He, S.G. Li, R.L. Lu, and L.H. Zhu (2000). Application of microsatellite markers for the seed purity examination of hybrid rice, Gangyou-22. Sheng Wu Gong Chengxue Bao.16: 211-214.

Michelmore, R.W.,I. Paran, and R.V. Kesseli (1991). Identification of markers linked to diseaseresistance genes by bulked segregant analysis: a rapid method to detect markers in specific genomic regions by using segregating populations. Proc. Natl. Acad. Sci. USA., 88: 9828-9832.

Mohammadi, S. and B.M. Prasanna (2003). Analysis of genetic diversity in crop plants-salient statistical tools and considerations. Crop Sci. 43:12351248.
Nene, Y.L. (1972). A survey of viral diseases of pulse crops in Uttar Pradesh. Final Technical Report. Res. Bull. No. 4, U.P. Agricultural University, Pantnagar, India. 1-91 pp.

Qazi, J.,M. Ilyas, S. Mansoor, and R.W. Briddon (2007). Legume yellow mosaic viruses: genetically isolated begomo viruses. Mol. Plant Pathol. 8: 343-348.

Rathi, Y.P. (2002). Epidemiology, yield losses and management of major diseases of Kharif pulses in India. In: Plant Pathology and Asian Congress of Mycology and Plant Pathology. University of Mysore, Mysore, India.

Singh, J.P. (1980). Effect of virus diseases on growth component and yield of mungbean and urdbean. Indian Phyto pathol. 8: 405-408.

Singh, S.K., G.R. Lavanya, K.V. Bhat, G.S. Babu, L.Arya, M. Verma, Z. Hussain, S. Roy, R.S. Rathi, and A.K. Misra (2012). Microsatellite markers revealed genetic diversity in mungbean mutant lines. Ind. J. Hill Fmg. 25: 38-43.

Tangphatsornruang, S.,P. Somta, P. Uthaipaisanwong, J. Chanprasert, D. Sangsrakru, W. Seehalak, and P. Srinives (2009). Characterization of microsatellites and gene contents from genomes hotgun sequences of mungbean (Vigna radiate (L.) Wilczek). BMC Plant Biol. 9(1): 137.

Wang, X.Q.S.W. Kwon, and J.K. Park (2012). Comparison of population genetic structures between Asian and American mungbean accessions using SSR markers. J. Agric. Sci. 4: $150-158$.

Yeh, F.C., R. Yang, T.J. Boyle, Z. Ye, and J.M. Xiyan (2000). Pop Gene 32, Microsoft windows-based free ware for population genetic analysis. Molecular biology and biotechnology center, University of Alberta, Edmonton, Canada. 